\title{
Bose-Fermi Mixtures in Optical Lattices
}

\author{
M. Centelles, M. Guilleumas, M. Barranco, R. Mayol, and M. Pi \\ Departament d'Estructura $i$ Constituents de la Matèria, Facultat de Física, \\ Universitat de Barcelona, Diagonal 647, 08028 Barcelona, Spain
}

\begin{abstract}
Using mean field theory, we have studied Bose-Fermi mixtures in a one-dimensional optical lattice in the case of an attractive boson-fermion interaction. We consider that the fermions are in the degenerate regime and that the laser intensities are such that quantum coherence across the condensate is ensured. We discuss the effect of the optical lattice on the critical rotational frequency for vortex line creation in the Bose-Einstein condensate, as well as how it affects the stability of the boson-fermion mixture. A reduction of the critical frequency for nucleating a vortex is observed as the strength of the applied laser is increased. The onset of instability of the mixture occurs for a sizeably lower number of fermions in the presence of a deep optical lattice.
\end{abstract}

\section{INTRODUCTION}

One of the most essential differences predicted between a superfluid and a normal fluid is the eventual appearance of quantized vortices in the former when set under rotation [1]. Not in vain, since the demonstration of the occurrence of Bose-Einstein condensation 2, 3, 4], the nucleation of vortices in dilute ultra-cold atomic gases in rotating traps has been instrumental to elucidate the superfluid properties of these systems and has attracted much experimental and theoretical research (see e.g. Refs. 5, 6, 7, 8, 9, 10, 11, 12, 13] and references quoted therein). The prominence of the study of vortices in atomic gases transcends the frontiers of the field itself, because topological defects are cornerstones in crossdisciplinary areas of physics. In turn, very recently, the experimentation with Bose-Einstein condensates in optical lattices is stimulating new avenues in the investigation of quantum coherence and interference phenomena.

In the present work we study some properties of a slowly rotating Bose-Einstein condensate (BEC) in a one-dimensional (1D) optical lattice 14]. We are motivated by the recent advances achieved in the experimental manipulation of rotating condensates [15, 16], on the one hand, and of condensates loaded into optical lattices [17], on the other hand. Rotating ultracold Bose gases in optical lattices are suitable systems to study quantum phenomena. In particular, the excitations, stability and dynamics of a vortex line in the superfluid regime have been recently addressed [18]. Concurrently, the physics of degenerate Bose-Fermi mixtures in optical lattices is attracting conspicuous attention. Theoretical progress is being made in the basic understanding of the system's quantum phase diagram and the superfluid to Mott-insulator transition [19], and experimentalists have already been able to prepare a degenerate mixture of $\mathrm{Rb}$ and $\mathrm{K}$ atoms in a tight optical lattice [20].

The aim of this work is twofold. Firstly, we wish to address the formation of vortices in a coherent array of Bose-Einstein condensates in a $1 \mathrm{D}$ optical lattice. In doing this, we will determine the dependence of the thermodynamic critical angular velocity $\Omega_{c}$ for vortex formation $[5,6]$ on the laser intensity. Secondly, we want to inves- tigate the effect of trapped fermions on $\Omega_{c}$. Attractive Bose-Fermi mixtures may experience collapse and thus, we will also discuss the impact of the optical lattice on the mechanical stability of the mixture.

The manuscript has been organized as follows. In Section \a short description of the mean field model for the present problem is given. Section III is devoted to the discussion of the numerical results for a rotating boson condensate confined by magnetic and optical traps. In Section IV we address the properties of a ${ }^{87} \mathrm{Rb}^{40}{ }^{40} \mathrm{~K}$ mixture loaded in an optical lattice. Our concluding remarks are laid in Section D Finally, a derivation of the virial theorem for the mixture in the 1D optical lattice is given in the Appendix.

\section{THEORY}

We consider a zero temperature mixture made of a ${ }^{87} \mathrm{Rb}$ Bose condensate (B) and a degenerate ${ }^{40} \mathrm{~K}$ Fermi gas (F). They are confined by the axially-symmetric external potentials of a harmonic magnetic trap and of a stationary periodic optical lattice modulated along the $z$-axis. The lattice is produced by a far detuned laser, which hinders the possibility of spontaneuous scattering and yields practically equal $\mathrm{Rb}$ and $\mathrm{K}$ optical potentials. The resulting potential for each kind of atom $q=B, F$ is

$$
V_{q}=\frac{1}{2} m_{q}\left(\omega_{q \perp}^{2} r^{2}+\omega_{q z}^{2} z^{2}\right)+\frac{V_{0}}{2} \cos (2 \pi z / d),
$$

where $m_{q}$ is the atomic mass and $r=\sqrt{x^{2}+y^{2}}$ is the radial variable of cylindrical coordinates.

The radial and axial frequencies of the harmonic trap are taken from a recent experiment [17]: $\omega_{B \perp}=2 \pi \nu_{B \perp}$ with $\nu_{B \perp}=74 \mathrm{~Hz}$, and $\omega_{B z}=2 \pi \nu_{B z}$ with $\nu_{B z}=11 \mathrm{~Hz}$ for ${ }^{87} \mathrm{Rb}$, while those for ${ }^{40} \mathrm{~K}$ are a factor $\left(m_{B} / m_{F}\right)^{1 / 2} \simeq$ 1.47 larger. The optical potential is determined by its period $d=2.7 \mu \mathrm{m} \mathrm{[17]}$ and depth $V_{0}=s E_{R}$, where $E_{R}=$ $\hbar^{2} \pi^{2} / 2 m d^{2}=h \times 80 \mathrm{~Hz}$ is the recoil energy and $s$ is a dimensionless parameter that provides the intensity of the laser beam.

For $3 \times 10^{5}$ atoms of ${ }^{87} \mathrm{Rb}$ confined in the harmonic trap, the condensate is cigar-shaped with a Thomas- 
Fermi length $L_{\mathrm{TF}}=84 \mu \mathrm{m}$, and a radius $R_{\mathrm{TF}}=6 \mu \mathrm{m}$. The superimposed optical potential splits the BEC over $L_{\mathrm{TF}} / d \sim 30$ wells. We shall be concerned with a range of laser intensities that ensures full coherence of the condensate across the whole system [18]. The criterion for the Mott transition [21] for the considered parameters leads to an estimate of a maximum lattice depth for the superfluid regime of $V_{0} \sim 150 E_{R} \sim h \times 12 \mathrm{kHz}$ [17]. Provided that the strength $V_{0}$ does not surpass largely this value, quantum tunneling between consecutive wells is still sufficient to retain coherence and one can apply the Gross-Pitaevskii theory for the order parameter to study the properties of the condensate.

Within the mean-field approach, neglecting $p$-wave interactions, the energy density functional that describes the boson-fermion mixture at zero temperature with a quantized vortex in the condensate along the $z$-axis has the form 22, 23.

$$
\begin{aligned}
\mathcal{E}(\mathbf{r})= & \frac{\hbar^{2}}{2 m_{B}}\left[\left(\boldsymbol{\nabla} n_{B}^{1 / 2}\right)^{2}+\frac{\kappa^{2}}{r^{2}} n_{B}\right]+V_{B} n_{B}+\frac{1}{2} g_{B B} n_{B}^{2} \\
& +\frac{\hbar^{2}}{2 m_{F}}\left[\frac{3}{5}\left(6 \pi^{2}\right)^{2 / 3} n_{F}^{5 / 3}+\frac{1}{36} \frac{\left(\boldsymbol{\nabla} n_{F}\right)^{2}}{n_{F}}\right] \\
& +V_{F} n_{F}+g_{B F} n_{F} n_{B}
\end{aligned}
$$

where $\kappa$ is the circulation quantum number, $n_{B}=|\Psi|^{2}$ is the condensate density, $n_{F}$ is the fermion density, and the fermion kinetic energy density has been written in the Thomas-Fermi-Weizsäcker approximation. The wave function of the boson condensate with a vortex line is $\Psi=\sqrt{n_{B}(r, z)} e^{i \kappa \phi}$, where $\phi$ refers to the azimuthal angle in the xy-plane. In this work we consider the cases $\kappa=0$ (non-rotating condensate) and $\kappa=1$ (condensate with a singly quantized vortex). As is known, vortices with more than one quantum of circulation will not exist in equilibrium [9]. The boson-boson and boson-fermion coupling constants $g_{B B}$ and $g_{B F}$ that enter Eq. (2) are written in terms of the $s$-wave scattering lengths $a_{B}$ and $a_{B F}$ as $g_{B B}=4 \pi a_{B} \hbar^{2} / m_{B}$ and $g_{B F}=4 \pi a_{B F} \hbar^{2} / m_{B F}$, respectively, with $m_{B F} \equiv 2 m_{B} m_{F} /\left(m_{B}+m_{F}\right)$.

The variation of $\mathcal{E}$ with respect to $n_{B}$ and $n_{F}$, under the constraint of given number of bosons $N_{B}$ and fermions $N_{F}$, yields two coupled Euler-Lagrange equations. Namely, a Gross-Pitaevskii equation for bosons with a term describing the boson-fermion interaction:

$$
\begin{gathered}
\left\{\frac{\hbar^{2}}{2 m_{B}}\left[-\Delta+\frac{\kappa^{2}}{r^{2}}\right]+V_{B}+g_{B B} n_{B}\right. \\
\left.+g_{B F} n_{F}\right\} n_{B}^{1 / 2} \\
=\mu_{B} n_{B}^{1 / 2}
\end{gathered}
$$

and a Thomas-Fermi-Weizsäcker equation for fermions [22, 23] which is increasingly valid the larger $N_{F}$ is:

$$
\begin{gathered}
\left\{\frac{\hbar^{2}}{2 m_{F}}\left[\left(6 \pi^{2}\right)^{2 / 3} n_{F}^{2 / 3}+\frac{1}{36} \frac{\left(\nabla n_{F}\right)^{2}}{n_{F}^{2}}-\frac{1}{18} \Delta\right]\right. \\
\left.+V_{F}+g_{B F} n_{B}\right\} n_{F}=\mu_{F} n_{F}
\end{gathered}
$$

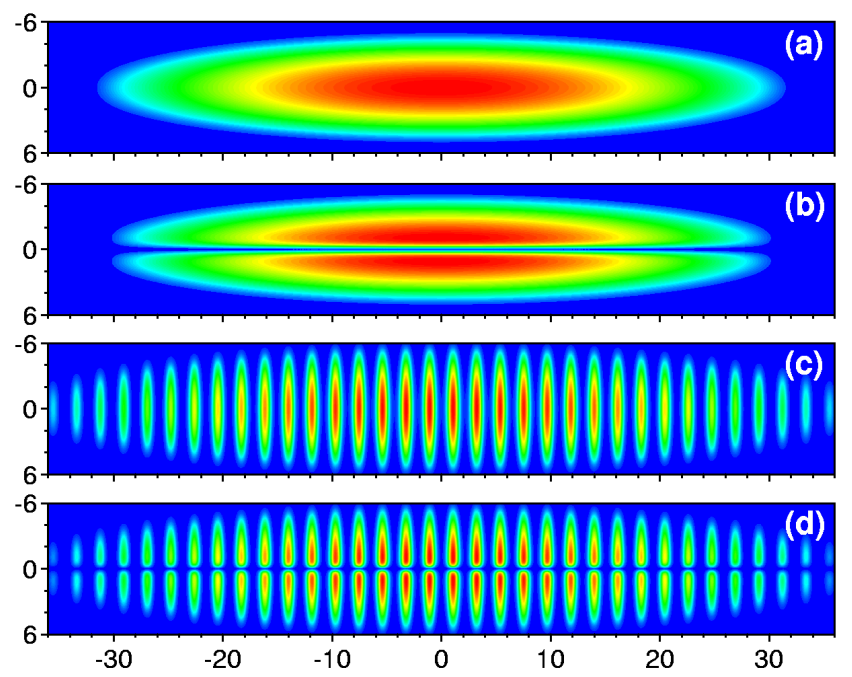

FIG. 1: (Color online) Contour plots of the ${ }^{87} \mathrm{Rb}$ condensate density in the $x z$-plane. (a) Condensate in the pure harmonic trap. (b) Same as (a) with a quantized vortex along the $z$ axis. (c) Same as (a) under an optical lattice with $V_{0} / h=$ $2.5 \mathrm{kHz}$. (d) Same as (c) with a quantized vortex along the $z$-axis. Distances are in units of the oscillator length $a_{B \perp}=$ $\sqrt{\hbar /\left(m_{B} \omega_{B \perp}\right)}=1.25 \mu \mathrm{m}$. In each panel the color scale ranges from deep blue at lowest densities to red at highest densities [these are $229(\mathrm{a}), 212(\mathrm{~b}), 394(\mathrm{c})$, and $\left.376 a_{B \perp}^{-3}(\mathrm{~d})\right]$.

where $\mu_{B}$ and $\mu_{F}$ are the chemical potentials of the boson and fermion atomic species, respectively. The ground state of the boson-fermion mixture is found with $\kappa=0$, while the vortical state is computed with $\kappa=1$. Starting from randomly sampled densities, we solve Eqs. (3) and (4) by means of the imaginary time method 24]. We use the adapted version of the virial theorem [23] to check the numerical convergence of the solution (see the Appendix).

\section{BOSON CONDENSATE LOADED INTO AN OPTICAL LATTICE}

We first consider a pure boson condensate, with $N_{B}=$ $3 \times 10^{5}$ atoms of ${ }^{87} \mathrm{Rb}$ as in the experiment of Ref. [17]. For the boson-boson scattering length we use the value $a_{B}=98.98 a_{0}\left(1 a_{0}=0.529 \AA\right)$ 25]. In Fig. 11 we show contour plots of the condensate density in the $x z$-plane, $n_{B}(x, 0, z)=|\Psi(x, 0, z)|^{2}$, for different situations. In (a) the condensate is confined only by the harmonic trap. In (b) the cigar-shaped condensate of (a) hosts a quantized vortex line along the $z$-axis. In (c) a $1 \mathrm{D}$ optical lattice with $V_{0} / h=2.5 \mathrm{kHz}$ splits the cigar-shaped condensate (a) into an array of multiple disk-like coherent condensates.

As shown in Ref. [26], certain aspects of the macroscopic properties and low-energy dynamics of a magnetically trapped BEC in a tight optical lattice can be understood in terms of a renormalized interaction coupling 

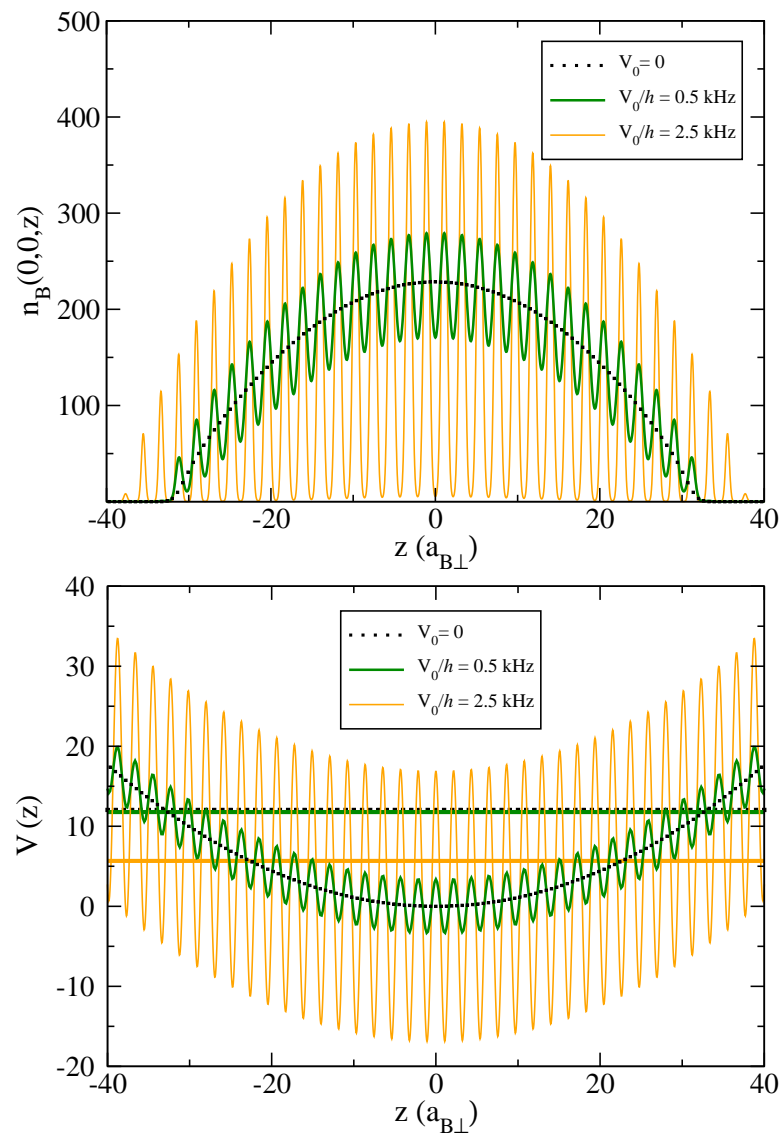

FIG. 2: (Color online) Top: Density profile of the condensate along the $z$-axis $n_{B}(0,0, z)=|\Psi(0,0, z)|^{2}$, in units of $a_{B \perp}^{-3}$, for the indicated values of the lattice depth $V_{0}$. Bottom: The $z$-dependence of the combined potential of the harmonic trap and the optical lattice, Eq. (1), in units of $\hbar \omega_{B \perp}$. The horizontal lines depict the location the chemical potential of the condensate boson cloud trapped in that potential.

constant $g_{B B}^{*}>g_{B B}$ and of an effective mass $m_{B}^{*}>m_{B}$ along the direction of the periodic optical potential. The increase of the radial size of the sample that one observes in panel (c) with respect to panel (a) is due to the increased repulsive effect of the boson-boson interaction when the system feels the optical lattice. It originates from the fact that the optical confinement produces a local compression of the gas inside each well 21, 26]. The axial size of the condensate also increases appreciably due to the combined effect of the repulsive interactions and the redistribution of atoms inside the potential wells.

In panel (d) of Fig. 1 the optical lattice is superimposed to the condensate with the vortex state (b). In a bulk superfluid, the size of the vortex core is of the order of the healing length $\xi=\left[8 \pi n_{B} a_{B}\right]^{-1 / 2}$, where $n_{B}$ is the bulk density. This expression holds for inhomogeneous superfluids taking for $n_{B}$ the local boson density in the absence of vortices [6]. Thus, the vortex core size is larger for the outer sites of the split condensate, where the density is smaller.

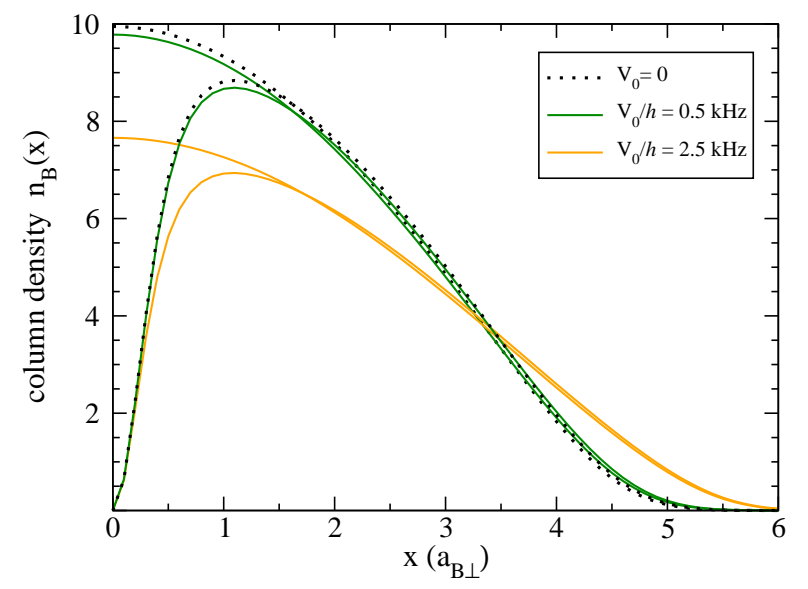

FIG. 3: (Color online) Column density $n_{B}(x)$, in units of $10^{3} \times a_{B \perp}^{-2}$, for different laser intensities. The column density of the condensate when it hosts a vortex line is also plotted.

In the upper part of Fig. 2 we show the evolution of the density profile of the condensate along the $z$-axis, $n_{B}(0,0, z)=|\Psi(0,0, z)|^{2}$, with the laser intensity. In the lower part of Fig. 2 we depict the total trapping potential felt by the atomic condensate. In the presence of a shallow 1D optical lattice, the condensate profile starts developing small oscillations around the profile at $V_{0}=0$ which follow the periodic optical potential, but its spatial extension is nearly not affected. When the laser intensity increases the system nearly splits into separate condensates. The effect of the local compression inside each potential well of the lattice, as well as the increase of the axial size of the system, can be clearly seen in Fig. 2

In Fig. 3 we plot the column density $n_{B}(x)=$ $\int d z n_{B}(x, 0, z)$ for the condensate in the harmonic trap alone and when the optical lattice is added. A deep optical lattice results in a visible reduction of the column density at the interior of the condensate and in an extended surface. Figure 3 also displays $n_{B}(x)$ for the condensate hosting a vortex line. In this case the quantized circulation around the vortex line pushes the atoms away from it.

The effect of the modification of the value of the lattice constant $d$ is illustrated in Fig. 4 In this figure we plot the computed density profile $n_{B}(0,0, z)$ of the boson atoms in the condensate for the same parameters as in Fig. [2 but for a larger $d(d=6 \times 2.7 \mu \mathrm{m})$.

The thermodynamic critical angular velocity $\Omega_{c}$ for nucleating a singly quantized vortex is obtained by subtracting from the vortex state energy $E_{1}$ in the rotating frame the ground-state energy $E_{0}$, i.e., $\Omega_{c}=\left(E_{1} / N_{B}-\right.$ $\left.E_{0} / N_{B}\right) / \hbar[5,6]$, and it provides a lower bound to the critical angular velocity [6]. In Fig. [5 we plot $\Omega_{c}$ as a function of the laser intensity for $N_{B}=3 \times 10^{5}$ and for $N_{B}=5 \times 10^{4}$. For a fixed number of condensate atoms, $\Omega_{c}$ decreases when $V_{0}$ increases, in agreement with the fact that the radial size of the system becomes larger in the presence of the optical lattice and the associated 


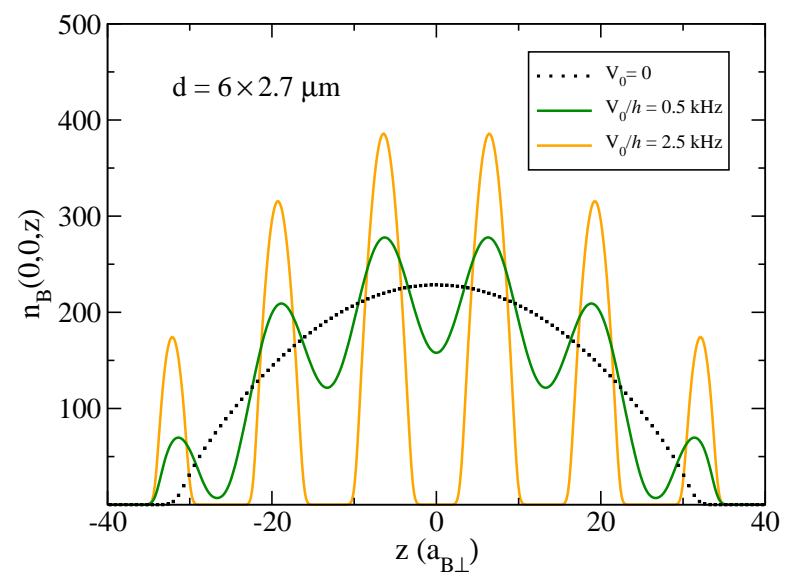

FIG. 4: (Color online) Density profile of the condensate along the $z$-axis $n_{B}(0,0, z)=|\Psi(0,0, z)|^{2}$, in units of $a_{B \perp}^{-3}$, for different laser intensities when the period of the optical lattice potential is increased to $d=6 \times 2.7 \mu \mathrm{m}$.

reduction of atoms along the symmetry axis (see the decrease of the column density $n_{B}$ at the origin in Fig. 3 with increasing $V_{0}$ ). In turn, the increase of the inertia of the condensate along the direction of the laser beam due to the larger effective mass $m_{B}^{*}[26]$ also contributes to the reduction of the critical angular velocity.

Assuming that the relative effect remains of the same order in experiment, Fig. 5 predicts a sizeable $24 \%$ reduction of $\Omega_{c}$ already with a laser strength $V_{0} / h=5 \mathrm{kHz}$ for both of the $N_{B}$ values. The effect is enhanced the shallower the magnetic trap is. A less elongated magnetic trap would favor the appearence of a vortex at a slower rotation. For example, the values of $\Omega_{c}\left(V_{0}=0\right)$ shown in Fig. 5 would be decreased by a 0.6 factor in a spherical trap with $\nu_{B z}=\nu_{B \perp}=74 \mathrm{~Hz}$. The relative reduction of $\Omega_{c}$ caused by $V_{0} / h=5 \mathrm{kHz}$ with respect to $V_{0}=0$ would still be of $20-23 \%$. As the thermodynamic $\Omega_{c}$ underestimates the actual critical frequency for vortex creation, dynamical calculations would be needed for a precise quantitative estimate of these changes.

Figure 5 suggests an experiment to test the dependence of the critical frequency for vortex nucleation on the intensity of the optical lattice, since it shows that, for a rotating condensate with a given $N_{B}$, a vortex line should nucleate at angular frequencies much lower than $\Omega_{c}\left(V_{0}=0\right)$ if a co-rotating deep $1 \mathrm{D}$ optical lattice is superimposed, whereas otherwise it would not. The experimental observation of this effect would provide a clear signature of the correctness of the physics predicted by the Gross-Pitaevskii theory for rotating condensates in optical lattices.

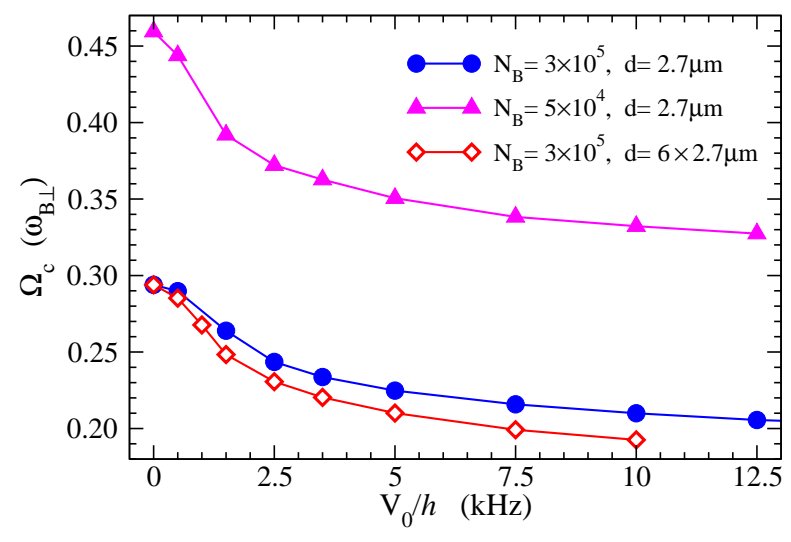

FIG. 5: (Color online) Critical angular velocity in units of $\omega_{B \perp}$ for nucleation of a singly quantized vortex line as a function of the laser intensity.

\section{ATTRACTIVE BOSON-FERMION MIXTURE LOADED INTO AN OPTICAL LATTICE}

With the purpose of assessing the effects of a trapped fermion cloud on the rotating condensate in an optical lattice, we consider next a ${ }^{87} \mathrm{Rb}^{-}{ }^{40} \mathrm{~K}$ mixture. It is characterized by a large attractive Bose-Fermi interaction that assists the sympathetic cooling of the fermionic species down to the degenerate regime. There are various values of the interspecies scattering length $a_{B F}$ published, depending on the type of experiment and techniques used [27, 28, 29, 30]. A recent measurement of $a_{B F}$ at JILA [30] has established a value $a_{B F}=$ $-250 \pm 30 a_{0}$, which we adopt here because of its reduced experimental uncertainties. Vortex states in ${ }^{87} \mathrm{Rb}^{-40} \mathrm{~K}$ mixtures confined by a harmonic potential have been recently addressed 23] (although $a_{B F}=-395 a_{0}$ 29] was employed). When a 1D optical lattice is switched on, the atomic species experience the trapping potential (11).

We have carried out calculations for a mixture with $N_{B}=3 \times 10^{5}$ and $N_{F}=1.5 \times 10^{5}$ atoms, and the same parameters as in Fig. [1 We assume that the Fermi component is in the normal-nonsuperfluid-but quantum degenerate phase, and consider that it is in a stationary state. This situation could be achieved experimentally by waiting long enough after the generation of the vortex in the condensate to let the drag force between bosons and fermions to dissipate.

We present in Fig. [6 contour plots of the boson $n_{B}(x, 0, z)$ and fermion $n_{F}(x, 0, z)$ densities when the condensate hosts a vortex line, for two laser strengths: $V_{0} / h=0.5(\mathrm{a}, \mathrm{b})$ and $2.5 \mathrm{kHz}(\mathrm{c}, \mathrm{d})$. The condensate and the fermionic cloud are modulated by the regular pattern of the optical lattice. The large mutual attraction between fermions and bosons makes the effective interaction between bosons less repulsive than in the pure BEC. Furthermore, the boson atoms induce an effective attraction between fermions counteracting the Fermi pressure. 

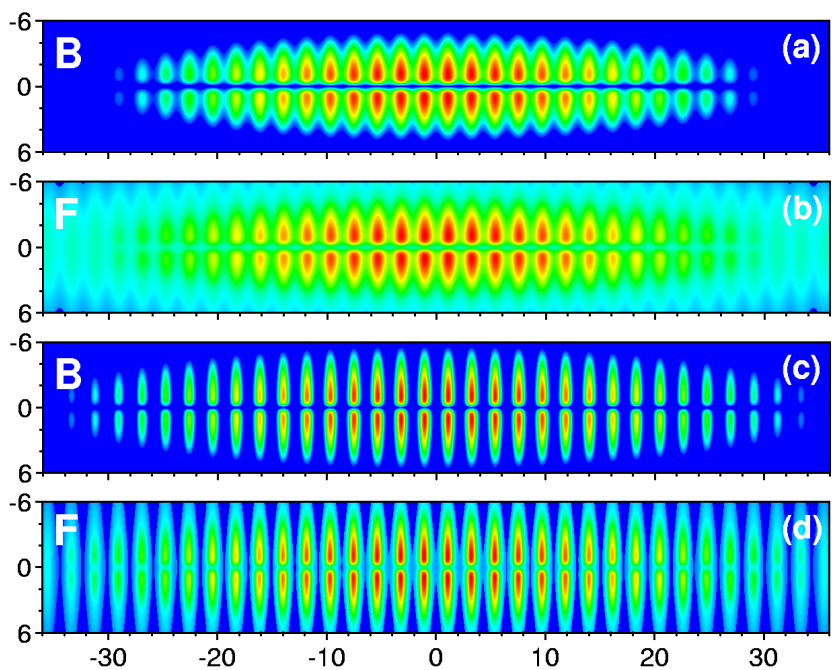

FIG. 6: (Color online) Contour plots of the condensate (B) and fermionic $(\mathrm{F})$ densities in the $x z$-plane for the ${ }^{87} \mathrm{Rb}$ ${ }^{40} \mathrm{~K}$ mixture, in the combined harmonic and optical lattice trap. The condensate hosts a quantized vortex. In (a) and (b) $V_{0} / h=0.5 \mathrm{kHz}$, whereas in (c) and (d) $V_{0} / h=2.5 \mathrm{kHz}$. Distances are in units of $a_{B \perp}$. In each panel the color scale ranges from deep blue at lowest densities to red at highest densities [these are 312 (a), 25 (b), 457 (c), and $37 a_{B \perp}^{-3}(\mathrm{~d})$ ].

As a result, the density of both species increases in the overlapping region, as if they were more strongly confined by the external potentials, and the condensate becomes more compact in space (compare Figs. 6] and 19). Still, the effect of the Pauli exclusion principle is notorious in Fig. [6] where the ${ }^{40} \mathrm{~K}$ cloud is seen to extend to larger distances than the ${ }^{87} \mathrm{Rb}$ atoms, both axially and radially.

The presence of a vortex along the $z$-axis in the condensate component is apparent in panels (a) and (c) of Fig. 6 as the boson density vanishes on the vortex line. It is interesting to note that the large attractive $a_{B F}$ leads to a visible depletion of the fermionic density on the $z$ axis as well, reminiscent of the bosonic vortex core. The effect should be directly observable experimentally. It is more evident in (d) where bosons and fermions are more tightly confined by the optical lattice.

In the present mixture we find that the presence of the quantum degenerate fermionic cloud does not change much the value of $\Omega_{c}$ compared to the pure BEC, nor its pattern against the optical lattice strength. Indeed, for all the $V_{0}$ values considered here the critical frequency for vortex appearence is raised by $\sim 10 \%$, due to the enhancement of the condensate density at the core caused by the fermion atoms. The impact of the fermion cloud on $\Omega_{c}$ is magnified in a deeper magnetic trap. For instance, if the same mixture was set in a trap with $\nu_{B z}=95 \mathrm{~Hz}$ and $\nu_{B \perp}=640 \mathrm{~Hz}$, that preserves the aspect ratio $\nu_{B z} / \nu_{B \perp}=0.15$ used in Fig. [6] the critical frequency $\Omega_{c}\left(V_{0}=0\right)$ would increase by $40 \%$ with respect to the $N_{F}=0$ case. In this squeezing magnetic trap, however, the effect of $V_{0}$ on $\Omega_{c}$ is negligible $(<2 \%)$.
A peculiar feature of the ${ }^{87} \mathrm{Rb}-{ }^{40} \mathrm{~K}$ system, that stems from the interplay of the moderately repulsive $a_{B}$ and the strongly attractive $a_{B F}$, is the existence of a mechanical stability limit beyond which the system cannot sustain more fermions. At high densities the strong interspecies attraction may overcome the Pauli pressure and drive the ultracold gas mixture into collapse. The phenomenon has been observed experimentally 28. This motivates us to address next the stability of the ${ }^{87} \mathrm{Rb}-{ }^{40} \mathrm{~K}$ system subject to an optical lattice. In fact, collapse is currently the focus of experimental attention because of the implications the instability has for constraining $a_{B F}$ [28, 29, 30], and also because there are prospects that a radially-squeezed ${ }^{87} \mathrm{Rb}^{40} \mathrm{~K}$ mixture at a density close to collapse must be able to form stable bright soliton trains [31].

If we keep $N_{B}$ fixed at $3 \times 10^{5}$ atoms as in the previous discussions, with $a_{B F}=-250 a_{0}$ we find that the boson-fermion mixture confined in the magnetic trap of Fig. [6 would be stable up to virtually arbitrary fermion numbers, consistently with the findings of Ref. 222. Increasing $\omega_{B \perp}$ prompts the occurrence of collapse. In a trap with $\nu_{B z}=95 \mathrm{~Hz}$ and $\nu_{B \perp}=640 \mathrm{~Hz}$, the $3 \times 10^{5}$ rubidium atoms are able to retain up to a maximum of $N_{F}^{\max }=2.6 \times 10^{5}$ potassium atoms, when the system collapses. We recall that the effect on $\Omega_{c}$ of $N_{F}=1.5 \times 10^{5}$ and $V_{0}$ for this squeezing trap has been discussed before.

Now, with $\nu_{B z}=95 \mathrm{~Hz}$ and $\nu_{B \perp}=640 \mathrm{~Hz}$, a decrease of $N_{F}^{\max }$ can be seen when a $1 \mathrm{D}$ optical lattice is added. It is related to the increase of the effective local compression of the atoms caused by the optical trap. Indeed, superimposing a $1 \mathrm{D}$ optical lattice with $d=2.7 \mu \mathrm{m}$ and $V_{0} / h=5 \mathrm{kHz}$ to this strongly confining harmonic trap, the ${ }^{87} \mathrm{Rb}^{40} \mathrm{~K}$ mixture with $N_{B}=3 \times 10^{5}$ can sustain up to $N_{F}^{\max }=7.5 \times 10^{4}$ fermions only, i.e., less than $30 \%$ of the $V_{0}=0$ value. The effect appears to be accessible to verification under present experimental conditions. It opens the possibility to study the collapse of a mixture for trapped fermion and boson numbers considerably smaller than in a pure harmonic trap.

\section{CONCLUSION}

Summarizing, we have determined the thermodynamic critical frequency for nucleating a quantized vortex in an array of coherent condensates within the Gross-Pitaevskii theory. Next we have studied a Bose-Fermi mixture trapped in a $1 \mathrm{D}$ optical lattice to examine the effect on the value of $\Omega_{c}$ of a fermionic cloud in the quantum degenerate phase. ${ }^{87} \mathrm{Rb}-{ }^{40} \mathrm{~K}$ mixtures may collapse and, in this regard, it has been shown that a 1D optical lattice can be an efficient degree of freedom to tune the onset of instability. Our analysis of the relative variation of $\Omega_{c}$ calculated by thermodynamic arguments may provide a useful information for future experiments. 


\section{ACKNOWLEDGMENTS}

We thank Dr. K. Bongs for valuable discussions. This work has been performed under Grants No. BFM200201868 from DGI (Spain) and FEDER, and No. 2001SGR00064 from Generalitat de Catalunya. M.G. thanks the "Ramón y Cajal" Program (Spain) for financial support.

\section{APPENDIX}

In this Appendix we make use of the principle of scale invariance to obtain the virial theorem for the energy functional of the boson-fermion mixture in an optical lattice. The virial theorem results from homogeneity properties of the kinetic and potential components of the energy of the many-body system with respect to a scaling transformation that preserves the normalization [32].

A normalized scaled version of the particle density, for either bosons or fermions, is

$$
n_{\lambda}(\mathbf{r})=\lambda^{3} n(\lambda \mathbf{r}),
$$

where $\lambda$ is an arbitrary scaling parameter. On account of (5), a straightforward manipulation shows that the various contributions to the energy from the functional (2) scale following the rules

$$
T_{\lambda}=\lambda^{2} T
$$

for the kinetic energy (including the boson vortex term if present),

$$
U_{H \lambda}=\frac{1}{\lambda^{2}} U_{H}
$$

for the harmonic potential terms, and

$$
U_{g \lambda}=\lambda^{3} U_{g} \quad \text { and } \quad U_{g_{B F} \lambda}=\lambda^{3} U_{g_{B F}}
$$

for the intra- and inter-species interaction terms. The contribution of the optical lattice transforms as

$$
\begin{aligned}
U_{\mathrm{opt}, \lambda} & =\frac{V_{0}}{2} \int d(\lambda \mathbf{r}) \cos \left[\frac{2 \pi(\lambda z)}{\lambda d}\right] n(\lambda \mathbf{r}) \\
& =U_{\mathrm{opt}}(\lambda d) .
\end{aligned}
$$

Hence, the total energy of the system scales as

$$
\begin{aligned}
E_{\lambda}= & \lambda^{2}\left(T_{B}+T_{F}\right)+\frac{1}{\lambda^{2}}\left(U_{H_{B}}+U_{H_{F}}\right) \\
& +\lambda^{3}\left(U_{g_{B B}}+U_{g_{B F}}\right) \\
& +U_{\mathrm{opt}_{B}}(\lambda d)+U_{\mathrm{opt}_{F}}(\lambda d) .
\end{aligned}
$$

And, finally, the stationarity condition of the energy against dilation leads to the virial theorem for the bosonfermion mixture:

$$
\begin{aligned}
0= & \left.\frac{d E_{\lambda}}{d \lambda}\right|_{\lambda=1} \\
= & 2\left(T_{B}+T_{F}\right)-2\left(U_{H_{B}}+U_{H_{F}}\right) \\
& +3\left(U_{g_{B B}}+U_{g_{B F}}\right) \\
& +\frac{1}{d}\left[\frac{\partial U_{\mathrm{opt}_{B}}}{\partial d}+\frac{\partial U_{\mathrm{opt}_{F}}}{\partial d}\right],
\end{aligned}
$$

where

$$
\begin{aligned}
\frac{1}{d} & {\left[\frac{\partial U_{\mathrm{opt}_{B}}}{\partial d}+\frac{\partial U_{\mathrm{opt}_{F}}}{\partial d}\right] } \\
& =\frac{V_{0}}{2} \int d \mathbf{r} \sin \left[\frac{2 \pi z}{d}\right] \frac{2 \pi z}{d}\left[n_{B}(\mathbf{r})+n_{F}(\mathbf{r})\right]
\end{aligned}
$$

Equation (11) is a useful tool to check the accuracy of the numerical solution of the coupled Gross-Pitaevskii and Thomas-Fermi-Weizsäcker equations. As a typical example, in our calculations of the mixture with $N_{B}=$ $3 \times 10^{5}$ and $N_{F}=1.5 \times 10^{5}$ atoms in an optical lattice of strength $V_{0} / h=2.5 \mathrm{kHz}$, from our converged solution we find that the rhs of Eq. (11) takes values $\sim 9(\sim-80)$ without (with) a vortex line, while $U_{H}$ is $\sim 6 \times 10^{6}$ and $U_{\text {opt }}$ is $\sim-5 \times 10^{6}$.
[1] R. J. Donnelly, Quantized Vortices in Helium II (Cambridge University Press, Cambridge, 1991).

[2] M. H. Anderson, J. R. Ensher, M. R. Matthews, C. E. Wieman, and E. A. Cornell, Science 269, 198 (1995).

[3] C. C. Bradley, C. A. Sackett, J. J. Tollett, and R. G. Hulet, Phys. Rev. Lett. 75, 1687 (1995).

[4] K. B. Davis, M. -O. Mewes, M. R. Andrews, N. J. van Druten, D. S. Durfee, D. M. Kurn, and W. Ketterle, Phys. Rev. Lett. 75, 3969 (1995).

[5] F. Dalfovo and S. Stringari, Phys. Rev. A 53, 2477
(1996).

[6] A. L. Fetter and A. A. Svidzinsky, J. Phys.: Condens. Matter 13, R135 (2001).

[7] J. J. García-Ripoll and V. M. Pérez-García, Phys. Rev. A 60, 4864 (1999).

[8] Ph. W. Courteille, V. S. Bagnato, and V. I. Yukalov, Laser Phys. 11, 659 (2001).

[9] C. J. Pethick and H. Smith, Bose-Einstein Condensation in Dilute Gases (Cambridge University Press, Cambridge, 2002). 
[10] L. Pitaevskii and S. Stringari, Bose-Einstein Condensation (Clarendon Press, Oxford, 2003).

[11] M. R. Matthews, B. P. Anderson, P. C. Haljan, D. S. Hall, C. E. Wieman, and E. A. Cornell, Phys. Rev. Lett. 83, 2498 (1999).

[12] K. W. Madison, F. Chevy, W. Wohlleben, and J. Dalibard, Phys. Rev. Lett. 84, 806 (2000).

[13] J. R. Abo-Shaeer, C. Raman, J. M. Vogels, and W. Ketterle, Science 292, 476 (2001).

[14] Fast rotating condensates hosting a large number of vortices are also currently investigated, see e.g. M. Tsubota, K. Kasamatsu, and M. Ueda, Phys. Rev. A 65, 023603 (2002); P. Engels, I. Coddington, P. C. Haljan, V. Schweikhard, and E. A. Cornell, Phys. Rev. Lett. 90, 170405 (2003); H. Zhai, Qi Zhou, R. Lü, and L. Chang, Phys. Rev. A 69, 063609 (2004); A. L. Fetter, B. Jackson, and S. Stringari, Phys. Rev. A 71, 013605 (2005).

[15] P. Rosenbusch, V. Bretin, and J. Dalibard, Phys. Rev. Lett. 89, 200403 (2002).

[16] V. Bretin, S. Stock, Y. Seurin, and J. Dalibard, Phys. Rev. Lett. 92, 050403 (2004).

[17] Z. Hadzibabic, S. Stock, B. Battelier, V. Bretin, and J. Dalibard, Phys. Rev. Lett. 93, 180403 (2004).

[18] J.-P. Martikainen and H. T. C. Stoof, Phys. Rev. Lett. 91, 240403 (2003); Phys. Rev. A 69, 053617 (2004); P. G. Kevrekidis, R. Carretero-González, G. Theocharis, D. J. Frantzeskakis, and B. A. Malomed, J. Phys. B: At. Mol. Opt. Phys. 36, 3467 (2003); A. B. Bhattacherjee, O. Morsch, and E. Arimondo, J. Phys. B: At. Mol. Opt. Phys. 37, 2355 (2004); J. W. Reijnders and R. A. Duine, Phys. Rev. Lett. 93, 060401 (2004).

[19] M. Lewenstein, L. Santos, M. A. Baranov, and H. Fehrmann, Phys. Rev. Lett. 92, 050401 (2004); M. Cramer, J. Eisert, and F. Illuminati, Phys. Rev. Lett.
93, 190405 (2004).

[20] G. Modugno, F. Ferlaino, R. Heidemann, G. Roati, and M. Inguscio, Phys. Rev. A 68, 011601(R) (2003).

[21] W. Zwerger, J. Opt. B: Quantum Semiclass. Opt. 5, S9 (2003).

[22] R. Roth, Phys. Rev. A 66, 013614 (2002).

[23] D. M. Jezek, M. Barranco, M. Guilleumas, R. Mayol, and M. Pi, Phys. Rev. A 70, 043630 (2004).

[24] M. Barranco, M. Guilleumas, E. S. Hernández, R. Mayol, M. Pi, and L. Szybisz, Phys. Rev. B 68, 024515 (2003).

[25] E. G. M. van Kempen, S. J. J. M. F. Kokkelmans, D. J. Heinzen, and B. J. Verhaar, Phys. Rev. Lett. 88, 093201 (2002).

[26] M. Krämer, L. Pitaevskii, and S. Stringari, Phys. Rev. Lett. 88, 180404 (2002).

[27] G. Ferrari, M. Inguscio, W. Jastrzebski, G. Modugno, G. Roati, and A. Simoni, Phys. Rev. Lett. 89, 053202 (2002).

[28] G. Modugno, G. Roati, F. Riboli, F. Ferlaino, R. J. Brecha, and M. Inguscio, Science 297, 2240 (2002).

[29] M. Modugno, F. Ferlaino, F. Riboli, G. Roati, G. Modugno, and M. Inguscio, Phys. Rev. A 68, 043626 (2003).

[30] J. Goldwin, S. Inouye, M. L. Olsen, B. Newman, B. D. DePaola, and D. S. Jin, Phys. Rev. A 70, 021601(R) (2004).

[31] T. Karpiuk, M. Brewczyk, S. Ospelkaus-Schwarzer, K. Bongs, M. Gajda, and K. Rzążewski, Phys. Rev. Lett. 93, 100401 (2004).

[32] For scaling relations and the virial theorem see, e.g., R. G. Parr and W. Yang, Density-Functional Theory of Atoms and Molecules (Oxford University Press, New York, 1989), and references therein. 